\title{
COMMENT
}

\section{Towards a stronger science of human plasticity}

\section{Ulman Lindenberger ${ }^{1,2,3}$, Elisabeth Wenger ${ }^{1}$ and Martin Lövdén ${ }^{1,4}$}

\begin{abstract}
People have been told that 'brain training' will make them not only better at playing certain games but smarter in general. However, evidence indicates that brain plasticity is specific to the acquired skill. The search for neural mechanisms of transfer of training-induced benefits needs to build on this evidence.
\end{abstract}

Plasticity can be defined as the brain's capacity to respond to experienced demands with structural changes that alter the behavioural repertoire ${ }^{1}$. It is crucial for the ontogeny of many organisms, including birds and mammals, and sculpts organisms into unique individuals. Plasticity may also ameliorate trajectories of cognitive decline in adulthood and old age ${ }^{2}$. Hence, the concept of a plastic human mind has attracted much scientific and public attention. The 'brain-training' industry nurtures the hopes of many people by promoting the idea that playing games will make them smarter, more alert and able to learn faster and better. Central to these claims are the concepts of generalization and transfer, according to which training enhances not only the trained skill but also performance on similar skills or even general cognitive processing capacity. If this were true, then training on a task involving a cognitive ability, such as working memory, would improve performance on many or all tasks that critically depend on that ability. The cumulative evidence to support these claims is weak. In relation to working-memory training studies, for instance, some meta-analyses report small but reliable positive transfer effects to other laboratory tasks of working memory, whereas other meta-analyses report average effects that do not differ reliably from null. Moreover, a recent review of the adult cognitive intervention literature has found little evidence for the assertion that brain training improves the everyday cognitive competence of adults and delays cognitive decline ${ }^{3}$.

In light of these sobering results, the time has come to critically reflect on extant 'brain-training' research efforts. We need to strive towards an understanding of human plasticity that is informed by principles of brain development, large-scale theories of the brain and neural accounts of skill acquisition that incorporate recent insights into the mechanisms and dynamics of experience-dependent plasticity ${ }^{4}$. The central question ought to be: what are the mechanisms of plasticity in the human brain? Claims about generalization and transfer need to be derived from answers to this question.
On the positive side, human plasticity is, clearly, not restricted to early development. Pretest-posttest comparisons have revealed grey-matter increases after several months of juggling training, intensive studying for medical exams, foreign language acquisition, spatial navigation training, playing video games and tracing with the non-dominant hand. Similar changes have been observed after 2 weeks of mirror reading, a few days of signature writing with the non-dominant hand and even after only two sessions of practice in a complex whole-body balancing task. Plastic changes in grey-matter volume can undoubtedly emerge quite rapidly in human adults.

The discovery of experience-dependent cortical plasticity across different age periods up to later adulthood most likely fuelled the optimism about improving human cognitive abilities through 'brain training'. However, the presence of a plastic brain response is not diagnostic for the presence of transfer and real-life benefits. Training often results in overlearned responses, and concomitant structural changes are likely to serve a specialized function. Most examples of neural plasticity that arises from cognitive stimulation are specific to the trained skill and show a narrow transfer gradient. The specificity of plasticity induced by skill training stands in marked contrast to other forms of environmental intervention, such as physical exercise. The latter can enhance processing capacity through mechanisms that broadly affect cognition, such as improved vascularization and the release of growth factors.

Evidence at ontogenetic and microgenetic timescales supports an 'overproduction-pruning' model of plasticity. The model posits an increase in the number of synapses at the beginning of the plastic episode, which is followed by experience-dependent selective stabilization of behaviourally relevant connections and the elimination of those connections that prove to be functionally irrelevant. Using two-photon microscopy and optogenetic tools, the overproduction-pruning sequence has been observed in behaving animals with unprecedented precision in recent years ${ }^{4}$. Plastic changes in the sensory 
and motor cortices are marked by the rapid formation of new dendritic spines, followed by a slower process of spine elimination, returning the overall number of spines close to pre-intervention levels. The dendritic spines that have been newly formed and retained in the course of a plastic episode show a remarkable degree of structural stability over time and may function as the physiological substrate for skill retention and reactivation. This process seems to be specific to the practiced skill, with different skills encoded in different dedicated sets of synapses.

Macroscopically, the overproduction-pruning model leads to the hypothesis that plasticity in the human brain is accompanied by an initial phase of grey-matter volume expansion followed by a period of volume renormalization. To test this hypothesis, we recently acquired up to 18 structural magnetic resonance images over a 7-week period while 15 right-handed young adults practiced non-dominant, left-hand writing and drawing ${ }^{5}$. After 4 weeks of practice, increases in grey-matter volume in both left and right primary motor cortices relative to a control group were observed; another 3 weeks later, these differences were no longer reliable. Time-series analyses showed that grey matter in both primary motor cortices expanded during the first 4 weeks and then partially renormalized, particularly in the right hemisphere, in the presence of continued practice and increasing task proficiency.

These theoretical and empirical observations suggest that the field may need a fundamental reorientation in research emphasis and design. Crucially, the pretest-posttest design, which implicitly equates plasticity with monotonic growth, should be augmented by studies that track the progression of training-induced structural changes. To narrow the gap to animal models, such studies can be conducted with training paradigms targeting sensory or motor cortices ${ }^{5}$. These studies can also make use of recently developed imaging modalities that help to clarify the nature of physiological, functional and neurochemical changes in the course of a plastic episode. In particular, multiple structural assessments need to be complemented by functional assessments of performance on the trained task. Task-related functional activations in cortical areas undergoing plastic reorganization are likely to increase during the initial period of cortical expansion, and decrease in the course of renormalization, when the pruning of new connections has led to sparser coding of task-relevant perception-action links. One may even speculate that the transient increase in task-related metabolic load at the beginning of a plastic episode gives way to a more efficient, metabolically less costly task representation at its completion.

Furthermore, age-graded differences in plastic change deserve to gain centre stage and need to be delineated through age-comparative studies. Cognitive development from childhood to early adulthood, for example, is marked by an increasing reliance on topdown control in the service of goal-directed behaviour. This shifting balance may facilitate some aspects of plastic change and hinder others. Relatedly, local plastic changes need to be studied in a global context. For example, the primary cortices form part of a structured, complex learning architecture. Networks that generate and monitor new behavioural routines and action sequences belong to this architecture and contribute to individual differences in skill acquisition. Higher-order brain regions, such as prefrontal and temporal areas, are likely to signal and keep track of the mismatch between experienced demands and the current range of functioning. We expect that reductions in mismatch due to increasing task proficiency may be accompanied by decreasing activations in these areas.

Finally, we need to explore the physiological basis of individual differences in plasticity. Given that the formation of new neural connections is metabolically costly, one can assume that individual differences in brain metabolism may predict differences in plasticity. Furthermore, individual differences in plasticity within specific domains, such as auditory pitch discrimination, are probably linked to pre-existing differences in brain anatomy, such as the morphology of the primary auditory cortex. Behavioural genetic evidence suggests that a considerable portion of individual differences in plasticity is associated with genetic differences. Here, candidate-gene or genome-wide approaches can be used to examine the unique and interactive effects of single-nucleotide polymorphisms on transmitter systems known to affect excitatory-inhibitory balance in primary cortical areas, such as glutamate, GABA and dopamine.

So, will 'brain training' ever make us smarter, more alert and able to learn faster and better? We do not know. On the basis of what we do know, any answer to this question is likely to vary by age, individual and functional domain. Perhaps the real question we should be asking is: how can skill acquisition trigger neural changes that affect general cognitive processing capacity? Two scenarios seem possible. First, skill training may refine elements of skill (for example, lead to a better percept) and indirectly enhance other cognitive functions that rely on these elements (such as processing operations involving that percept). Second, training the skill may directly improve some function of general utility, through neural mechanisms that remain to be discovered. Research targeting these scenarios will contribute to a stronger science of human plasticity, perhaps paving the way for more-effective 'brain-training' tools. In the meantime, taking up physical exercise may be the better way to go.

\footnotetext{
Lövdén, M. et al. A theoretical framework for the study of adult cognitive plasticity. Psychol. Bull. 136, 659-676 (2010) Lindenberger, U. Human cognitive aging: corriger la fortune? Science 346, 572-578 (2014).

3. Simons, D. J. et al. Do "brain-training" programs work? Psychol. Sci. Public Interest 17, 103-186 (2016).

4. Hübener, M. \& Bonhoeffer, T. Neuronal plasticity: beyond the critical period. Cell 159, 727-737 (2014).

5. Wenger, E. et al. Repeated structural imaging reveals nonlinear progression of experience-dependent volume changes in human motor cortex. Cereb. Cortex. http://dx.doi.org/10.1093/cercor/ bhw141 (2016).
}

Acknowledgments

The authors thank L. Carstensen, J. Delius, S. Kühn, U. Mayr and N. Raz for valuable discussions, and the Max Planck Society for continued support.

Competing interests

The authors declare no competing interests. 